\title{
A COMPARISON OF CHARGES FOR CONTINUOUS AMBULATORY PERITONEAL DIALYSIS AND CENTER HEMODIALYSIS
}

\author{
Dean G. Smith* and John R. C. Wheeler \\ Department of Health Services Management and Policy. The University of Michigan. 1420 \\ Washington Heights. Ann Arbor. MI 48109-2029, U.S.A \\ (Receired in retised form 29 . April 1988)
}

\begin{abstract}
Use of continuous ambulatory peritoneal dialysis (CAPD) is increasing, and it is being promoted as a less expensive alternative to center hemodialysis (CHD). The debate over the relative charges for CAPD and CHD cannot generally be answered without considering the relationship between modality selection and patient characteristics. When selection and patient characteristics are accounted for, the difference between annual charges for CAPD and CHD patients is insignificant, in part because of the current payment system. The analysis suggests that patients using CHD may have lower charges than if they were using CAPD; similarly, patients using CAPD may have lower charges than if they were using CHD. Charges during CAPD training are lower than CHD or CAPD charges. Charges during the transition between CHD and CAPD tend to be higher than either CHD or CAPD due to additional hospitalizations. Estimated results suggest that encouraging current CHD patients to transfer to CAPD (or vice versa) may not have the desired effect of reducing chatges.
\end{abstract}

Continuous ambulatory peritoncal dialysis Charges End-stage renal disease Ilemodialysis

\section{INTRODUCTION}

Since the introduction of continuous ambulatory peritoneal dialyses (CAPD) in 1979, it has become a common alternative to center hemodialysis (CHD) and other modalities of treatment for end-stage renal disease (ESRD). CHD is still the treatment of choice for $80 \%$ of ESRD patients on dialysis, but the use of CAPD has been increasing and currently stands at over $14 \%$ of ESRD patients on dialysis [1,2].

The rapid increase in the use of CAPD has prompted debates over the relative effectiveness and cost of CAPD vs other modalities of treatment, especially CHD [3-8]. Some of the advantages of CAPD are reductions in anemia and hypertension and greater freedom of movement. But there are also the disadvantages of an increase in the risk of peritonitis and other

*Author for correspondence.

Supported by the Health Care Financing Administration (Grant No. 14 P 98372/5-04). catheter complications and a reduction in opportunities for medical supervision [9-11]. The net effect of these advantages and disadvantages has not been fully determined, but it is thought to vary depending on the characteristics of the patient.

Government reimbursement regulations attempt to encourage the use of CAPD, based on the belief that it is less costly than CHD [12]. Earlier studies have suggested that the cost of CAPD is somewhat less than the cost of CHD. but results have not been conclusive. Since the major disadvantage of CAPD is the risk of catheter complications, which may require hospitalization, many reports have focused on the relative use of inpatient hospital services. A higher number of hospitalizations for CAPD as compared to CHD patients was found by Bovbjerg et al. [3] and Carlson et al. [4]. However, no significant difference in the mean number of hospitalizations between patients on these two modalities was found by Manninen $e t$ al. [7] or Prowant et al. [8] 
Prowant et al. found hospital charges to be insignificantly lower for CAPD but found total charges to be significantly lower. However, this study included only 46 patients at one center and was unadjusted for patient characteristics. Evans et al. [5] found inpatient hospital reimbursement to be lower and total reimbursement to be lower for CAPD as compared to CHD, after adjusting for patient characteristics, but these differences were insignificant.

This paper reports on the relative Medicare allowable charges for CAPD and CHD for a sample of ESRD patients from the Michigan ESRD Study. This study examines ESRD charges in a more complex manner than have previous authors. The results are also more complex, but provide a more meaningful examination of charges. Two important differences from the previously cited work are the more explicit definition of modality employed and the consideration of the effects of patient characteristics on the selection of modality.

\section{MATERIALS AND METHODS}

Data for this investigation come from the Health Care Financing Administration (HCFA), through the Urban Institute. Data for 1982-1984 were requested for all adult Michigan patients with onset of ESRD from 1981-1983. The Urban Institute identified Michigan patients and prepared data sets from the various Medicare ESRD Program files. Five data sets were received, each corresponding to a different part of the Medicare data system. Four data sets, patient identification, medical evidence, transplant, and quarterly dialysis records, come from the ESRD Program Management and Medical Information System (PMMIS). The fifth data set, containing charge data, comes from HCFA provider billing records. Matching data from all sources resulted in 2292 complete observations and a sample of $1446(65.3 \%)$ complete observations on patients with CAPD, CHD or a combination. The remaining 796 observations pertain to patients with all other forms of treatment, including transplantation. All patients receiving tansplants at any point in time were classified as transplant patients, irrespective of their use of CHD or CAPD during the study period. Hence, the analyses presented in this paper are based on all adult Michigan patients using CAPD or CHD about whom complete data are available for any amount of time within the 3-year study period.

\section{Charges}

Charges are measured as Medicare allowable charges. These are the charges which serve as the basis for Medicare payments to providers for services rendered. In simplest terms, Medicare pays approximately $80 \%$ of allowable charges. Actual Medicare payments for dialysis services are based on predetermined fixed rates which are identical for CHD and CAPD patients. Prior to August 1983, renal physicians received monthly capitations under either the "initial method," which varied payment by patient treatment location and allowed additional fee-for-service charges, or the "alternative reimbursement method," which paid a fixed monthly payment which also varied payment by patient treatment location. Since August 1983, physicians and facilities have been paid monthly capitation payments which are the same for CHD and CAPD patients. For both dialysis treatments and physician services, Medicare Part B payments are set at $80 \%$ of the predetermined rates. Hospital payments are based on Medicare Part A allowable charges, which since 1983-1984 have been based on predetermined rates by diagnosis-related group (DRG). Because the deductibles for both Parts $A$ and $B$ are small relative to total charges, the correlation between Medicare allowable charges and Medicare expenditures for ESRD patients is nearly perfect $(r=0.98)$ and Medicare expenditures are approximately $80 \%$ of total charges. In this analysis, total charges are used as the measure of charges. Total charges $(100 \%)$ include payments made by Medicare $(80 \%)$ and payments by, or on behalf of, the patient $(20 \%)$, and are a reasonable estimate of total payments for Medicare covered services.

Our measure of charges does not include payments made by the patient or on the patient's behalf for non-covered medical services or other expenses associated with the receipt of services. The use of home health aides and travel costs are examples of noncovered services and other expenses, respectively. For current policy purposes, it is perhaps most relevant to focus on payments for covered services, and in particular on Medicare allowable charges for services. For future policy changes, understanding the variation in both total charges and total costs among treatment modalities would be useful. 
To the extent that Medicare payment rules succeed in setting allowable charges (or their fixed reimbursements) close to the costs of production, our measure of charges is an approximation of the production costs of ESRD treatment. Held and Pauly [13] have argued that because of the reimbursement rules and the incentives for competition on the basis of amenities, observed production costs are likely to exceed the minimum potential costs. The actual relationship between costs and charges has not been investigated and is an important area for future research.

\section{Selection}

The process of ESRD treatment is not typically the selection and subsequent use of a single, well-defined modality. Usually, patients begin treatment with CHD and then either seek a transplant or consider alternative forms of dialysis, although selecting CAPD as the initial modality is becoming more common. Medicare payment rates are currently identical for CAPD and CHD. However, reimbursement begins in the first month of treatment for patients selecting CAPD or CAPD training (and for transplant patients), and not until the third month after onset of ESRD for patients selecting CHD. Therefore, CAPD is being encouraged as an initial modality of treatment by the difference in Medicare payments of approximately U.S. $\$ 6000$. For patients already Medicare qualified or otherwise insured, this financial incentive is less important than for those patients without insurance.

The definition of modality provided by Smith and Wheeler [14] includes the "steady-state" (unchanged during the year) use of CHD and CAPD, "training" modalities and "transitional" modalities (changes during the year). All CAPD patients in this sample have spent some time in CAPD training (CAPDTR) and/or a combination of CAPD and CAPDTR (CAPDCOMB), and some have spent time in a transitional stage between CHD and CAPD (CHD-CAPD). Most patients on CHD have not changed from other treatments, although some have changed from CAPD and have spent time in a transitional stage between CAPD and CHD (CAPD-CHD).

The seletion of a particular modality, while not deterministic, is not completely random. Patients along with their physicians select that modality which is believed to be the most appropriate form of treatment, given their condition. No formal criteria have been followed for CHD and CAPD selection, as has been suggested for cancer patients by Simes [15] and cadaver kidney recipients by Starzl et al. [16] but the data suggest systematic selection of modalities. Cost may or may not be a direci determinant of modality selection, but relative costs are influenced by the modality selection process.

For example, suppose there were two patients. the younger with ESRD caused by hypertension and the older with ESRD caused by glomerulonephritis. While the younger patient might very well have lower costs using CAPD, the older might be prone to complications or have difficulty with the process of performing the tasks associated with CAPD, and as a result have lower costs using CHD. One could not conclude that one type of treatment was less costly than the other unless patient characteristics and selection were included in the analysis.

The characteristics of the patients observed in this study are presented in Table 1. CHD and CAPD patients in this sample tend to be similar in age, gender and time since onset of ESRD, but patients on CAPD, in training, or in transi-

Table 1. Patient characteristics by modality

\begin{tabular}{|c|c|c|c|c|c|c|}
\hline & \multicolumn{2}{|c|}{ Steady-state } & \multicolumn{2}{|c|}{ Training } & \multicolumn{2}{|c|}{ Transition } \\
\hline & $\mathrm{CHD}$ & CAPD & CAPDTR & САРCOMB & CHD-C $\triangle P D$ & CAPD CHD \\
\hline Age $(y r)$ & 58.2 & $52.3^{*}$ & 55.5 & $50.5^{*}$ & 54.8 & $48.0^{*}$ \\
\hline Sex (male) & 46.5 & $57.8^{*}$ & 49.5 & 52.2 & 44.1 & 37.5 \\
\hline Race (white) & 56.0 & $90.0^{*}$ & $86.6^{*}$ & $89.1^{*}$ & $88.2 *$ & $79.2^{*}$ \\
\hline Time since onset $(\mathrm{yr})$ & 2.0 & 1.9 & 2.1 & 2.0 & 2.2 & 2.1 \\
\hline \multicolumn{7}{|l|}{ Primary disease $(\%)$ : } \\
\hline Diabetes & 21.5 & 16.7 & 21.0 & 19.6 & 11.8 & 33.3 \\
\hline Glomerulonephritis & 22.4 & 26.7 & $33.9^{*}$ & $34.8^{*}$ & 26.5 & 29.2 \\
\hline Hypertension & 33.1 & 21.1 & 29.0 & $19.6^{*}$ & 23.5 & 25.0 \\
\hline Other & 23.0 & $35.6^{*}$ & $16.1^{*}$ & 26.1 & $38.2^{*}$ & 12.5 \\
\hline Observations & 1106 & 90 & 186 & 46 & 34 & 24 \\
\hline
\end{tabular}

*Indicates a significant difference from steady-state CHD (first column). $p<0.05$. two-tailed test. 
tion are much more likely to be white than CHD patients $(p<0.05)$. The porportion of nonwhites on CAPD $(10 \%)$ is lower than the proportion on CHD (44\%) and is reflective of differences in treatments by race which have been frequently noted and criticized in the literature [17]. Reasons for the differences in modality by race in this sample are not explainable on the basis of other available data on patient characteristics.

Hypertension is the leading primary disease causing ESRD among CHD patients, with the other diseases being evenly distributed. Glomerulonephritis tends to be the leading primary disease causing ESRD among CAPD, training and transitional patients, although there are no clear patterns of primary diseases within these groups of patients. Comparing the incidence of primary diseases across modalities, there are some significant differences, particularly in the incidence of diabetes, but again, there are no clear patterns.

\section{Estimation}

To adjust for modality selection and differences in patient characteristics, a two-part estimation technique is employed. Failure to account for selection would result in biased estimates of charges by modality [18]. In the first part, a self-selection probit model is estimated for each modality. Individual probit models for the selection of each modality are estimated using patient characteristics (age, sex, race, time since the onset of ESRD and primary disease) as determinants of selection. A potentially important limitation of this study is the lack of additional patient and physician data which might more exactly determine selection. It has been shown that there are patterns of physician treatment recommendations which are independent of the above listed patient characteristics [19].

The probit model estimations permit the calculation of an additional variable which, essentially, is an estimate of the likelihood that a particualr modality will be selected by a patient. The calculation of these variables uses the inverse of the Mill's ratio which takes the form, $R_{i j}=\phi\left(z_{i j} \mathbf{G}_{i}\right) /\left(\Phi\left(z_{i j} \mathbf{G}_{i}\right)\right.$, where $R$ is the ratio for the $i$ th individual using the $j$ th modality, $\phi$ and $\Phi$ are the probability and cumulative density functions for the standard normal distribution, respectively, $z$ is the matrix of independent variables and $\mathbf{G}$ is the vector of coefficients from the probit model estimations.
In the second part, the variable calculated in the first part $(R)$ is used as a covariate along with other patient characteristics (age, sex, race, primary disease causing ESRD, year of observation and length of time observed) in a multivariate ordinary least squares regression of the logarithm of annual charges. Logged charges are used to avoid the problems associated with extreme values which are frequently encountered in health care charge data [20]. Observations of charges of less than U.S. $\$ 500$ for more than one quarter of a year and charges in excess of U.S. $\$ 200,000$ are considered to be gross outliers and are not included in the final sample of 1496 . Inclusion of these 78 outliers $(5 \%)$, however, only affect the variances of final results and not the mean values.

Standardized values of patient characteristics for each modality are used in the estimated equations to determine the charges of ESRD treatment by modality. This approach is described in general by Maddala [21] and in terms of specific examples in Heckman [18] and Lee [22]. The standardized value for length of time of observation is 12 months for all modalities. Although some observations are for periods of time less than a full year, in particular those for training and transition between modalities, all estimates are annualized. The estimates of training and transition modalities must therefore be used with caution, as the charges associated with training and transition are actually for relatively short periods of time.

Since charges are estimated in logs, estimated standard deviations are also in logs. Standard deviations are therefore log-linear rather than linear and simple confidence intervals are not meaningful. Instead, $p$-values of two-tailed tests are presented for comparisons among estimates of charges.

The statistical analysis was conducted using the Michigan Interactive Data Analysis Systems (MIDAS), a program capable of handling the probit and multivariate least squares regression analyses required by this two-part technique.

\section{RESULTS}

Table 2 presents estimates of the costs of ESRD treatment by modality for patients with different characteristics. The columns reflect the estimated charges of patients with those characteristics. For example, under the column labeled "CHD," the entries indicate the estimate of what the charges would be for patients similar 
Table 2. Estimated treatment charges by modality and patient characteristics (constant U.S. \$1983)

\begin{tabular}{lccc}
\hline \multicolumn{3}{c}{ Patient characteristics } \\
Modality & Actual & CHD & CAPD \\
\hline Steady-State & & & \\
$\quad$ CHD & 23,344 & 23,344 & 22,688 \\
$\quad$ CADP & 21,429 & 25,915 & 21,429 \\
Training & & & \\
$\quad$ CAPDTR & $18,079^{*}$ & $15.232^{* \dagger}$ & $12,308^{* \dagger}$ \\
$\quad$ CAPDCOMB & $17.360^{*}$ & $13.664^{*+}$ & $11,316^{* \dagger}$ \\
Transition & & & \\
$\quad$ CHD -CAPD & 25.982 & $43.826^{* \dagger}$ & $30.094^{* \dagger}$ \\
$\quad$ CAPD CHD & 30.773 & $32,415^{*}$ & 26.792 \\
\hline
\end{tabular}

*Indicates a significant difference from steady-state CHD (first row), $p<0.05$, two-tailed test.

†Indicates a significant difference from steady-state CAPD (second row), $p<0.05$, two-tailed test.

to those currently on CHD if they were treated using each of the six modalities. Correspondingly, the rows reflect the estimated charges for treatment by each modality: for patients actually receiving that treatment, and for patients with the characteristics of those receiving CHD and CAPD. This table could be "completed" by listing additional columns for each of the training and transitional modalities. Only the steadystate use of CHD and CAPD are listed since a patient is not trained or in transition for a long period.

Patients with CAPD characteristics using CAPD have lower average annual charges than patients with CHD characteristics using CHD. Although the difference is not significant $(p=0.28)$, it is consistent with the observation that CAPD is less expensive than CHD. However. while it may be less expensive to treat patients with CAPD characteristics using CAPD, it is not necessarily less expensive to treat patients with other characteristics using CAPD.

Patients with CHD characteristics incur charges averaging U.S. $\$ 23,344$ using CHD. Estimated charges for these same CHD patients and U.S. \$25.915 if they were using CAPD. This difference is not statistically significant $(p=0.19)$, but it suggests an appropriate selection of CHD patients in the use of CHD. Patients with CAPD characteristics generate charges of U.S. \$21.429 using CAPD. Estimated charges for these same CAPD patients are U.S. \$22,688 using CHD. Once again this difference is not significant $(p=0.47)$. but it indicates an appropriate selection of CAPD patients in the use of CAPD.
Actual and estimated charges for all patients using the training for CAPD are significantly lower than the steady-state use of either CHD or CAPD. On the other hand, annualized charges during transition from CHD to CAPD and from CAPD to CHD are higher than the steady-statc use of cither CHD or CAPD. Actual transition charges, the charges for those patients actually making a change of modality. are higher going from CAPD to CHD than from CHD to CAPD. Estimated charges for CHD and CAPD patients are higher going from CHD to CAPD than from CAPD to CHD. This seemingly paradoxical result is discussed in the next section.

Not unexpectedly, different variables are important in determing the likelihood of the selection and the costs of different modalities. (Tables of probit and ordinary least squares regressions are not presented, but are available from the authors upon request.) Age and race are consistently significant in the selection of modalities of treatment, but are infrequently significant in the determination of charges. Year of observation and length of time observed are significant factors in the determination of changes in charges over time, but not of differences in charges across modalities. Race, time since the onset of ESRD, and primary disease causing ESRD are only occasionally significant factors in determining differences in charges across modalities.

While there were several changes in the ESRD payment system and specific rates during the study period, including the introduction of prospective payment for hospitalizations, the relationships described in Table 2 did not vary significantly or substantively using estimates of each year individually. Any effects on relative charges due to changes in the payment system were not observed.

Some variables which might be significant factors in determining selection and/or charges, such as complications, secondary illnesses and facility type, are not available in these data. The addition of more data on patient characteristics would certainly increase the validity of the reported results. Without additional data the results presented below must be considered preliminary

\section{DISCLSSION}

The relative charges of CAPD and CHD are examined using a more explicit definition of 
modality, statistical correction for modality selection and statewise data from the Michigan ESRD Study. The estimated difference between annualized charges for CHD and CAPD patients is positive, but insignificant. When selection and patient characteristics are considered, estimated charges suggests that, in terms of charges, patients appropriately select modalites. On the basis of this result, a general shift of patients from CHD to CAPD or vice versa would be unlikely to reduce Medicare allowable charges.

Although this analysis more completely examines costs than previous studies, the simple differences in adjusted CHD and CAPD costs are not the end of the comparison. The use of CAPD also implies the use of CAPDTR and perhaps CAPDCOMP, CHD-CAPD and/or CAPD-CHD, and several patients changed from one modality to the other. Therefore, the full charge for changing modality must take into account the charges incurred with the period of change. The importance of the annualized charges for training and transition, however, should not be exaggerated. The periods of time in training or in a transitional stage between modalities are typically quite short (a few weeks at most) and do not greatly influence total charges over long period of time.

The periods of time on CAPDTR and CAPDCOMP are typically associated with lower costs than either CHD or CAPD. This result is contrary to expectations since there are start-up costs associated with training and changing modalities. However, patients typically elect to begin training when their conditions are stable and their health is otherwise good. The lack of other adverse health conditions and the careful supervision during training result in lower charges. However, it is not the case that patients could elect to be trained permanently as a way to minimize charges.

The switching of modalities other than through training is associated with higher charges for both CAPD and CHD patients. The result that actual charges are higher for the CAPD-CHD transition than for the CHDCAPD transition was expected. Complications with CAPD and the possibility of failure of this modality have been noted in the literature. The result that estimated charges are higher for the CHD-CAPD transition than for the CAPD-CHD transition for both CHD and CAPD patients is somewhat paradoxical. The reason for this result is that the patients actually making the transitions differed in terms of their characteristics from the average CHD and CAPD patients. The most important of these characteristics is the presence of diabetes as the primary disease causing ESRD. Diabetics with ESRD have average charges which are higher than non-diabetics. Since the actual transition charge estimates for CHD-CAPD and CAPD-CHD have as a basis rates of diabetes of 11.8 and $33.3 \%$, respectively, CAPD CHD tends to be associated with higher charges. The standardization of the rates of diabetes at 21.5 and $16.7 \%$ for CHD and CAPD, respectively, is the cause of the reversal in estimated charges. This result should be taken with caution since it is based on relatively small numbers of patients making transitions.

Transition period in this sample were also frequently associated with a hospitalization, indicating perhaps that a change of modality was required because of the failure of a course of treatment. Only 4 of the 58 patients making transitions ( 2 in each direction) did not have an episode of hospitalization. Voluntary transfers may very well not result in higher charges. The reason for changing modality (required vs voluntary) is not available and should be explored in future investigations.

The number of hospitalizations for patients on each modality using their actual characteristics are shown in Table 3. The differences between the steady-state use of a modality and training are small and insignificant. However, the transitions are associated with an average of one more admission, and the differences for noted combinations are significant $(p<0.05)$. No data were available on hopsital days.

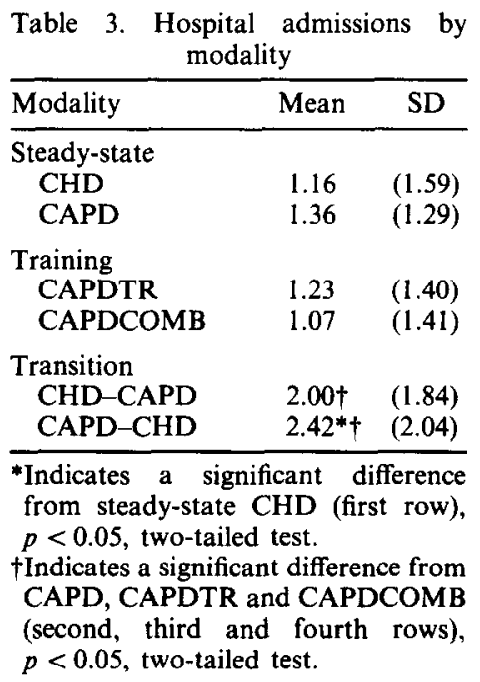


The difference between these hospitalization results and those of previous authors is most likely accounted for by the difference in the definition of modality. Other studies have not separated steady-stated use of well-defined modalities from training and transitional period. Since it is the transitional modalities which tend to have greater than the average number of admissions, the modality in which these transitions are included. CHD or CAPD. will be observed to have the higher rate of hospitalization.

A major result of this paper, that Medicare allowable charges for CHD and CAPD are very similar, can be largely attributed to the Medicare ESRD payment system which in recent years has established similar facility and physician reimbursement rates for CHD and CAPD. The principal conclusion of this paper must therefore be restated. It is that. given the current payment system, a shifting of patients from CHD to CAPD (or vice versa) is unlikely to result in a reduction in changes or Medicare expenditures. What we do not know is whether such shifts are likely to reduce the treatment costs to the provider. Research on this question needs to be done. But we argue strongly that this research must employ methods described in this paper; i.e. research on the relative costliness of ESRD modalities must account for the effects of patient characteristics on modality selection and costs

The current Medicare ESRD payment system is based on the assumption that CAPD costs are lower than CHD costs. Equal payments for CHD and CAPD are intended to give providers more profit from the presumably less costly CAPD and therefore to encourage a shift from CHD to CAPD. We believe that this policy is flawed in two respects. First, it is not based on a knowledge of the relative costs of modalities for particular patients. Second, the setting of equal prices for the two modalities means that any cost savings associated with a shift to CAPD are fully appropriated by the providers: the government and the patient do not benefit at all.

If it can be determined that. for patients with particular characteristics. one modality is less costly than the other. then the government should reconsider its payment system. Stronger incentives for the use of the most appropriate and least costly modality should be instituted. Such an incentive system might involve continuing the current physician payment system, which pays the same capitation for both $\mathrm{CHD}$ and CAPD, but altering the facility payment system to reflect the relative production costs. Such a system might leave the physician financially indifferent, but give the patient the incentive via the $20 \%$ coinsurance rate, to determine the least costly modality.

Finally, the scientifically most appropriate way to compare costs and control for patient characteristics and selection is to conduct a prospective study which randomly assigns patients to modalities of treatment. However. such an experiment is clinically inappropriate if modalities are indeed selected so as to maximize health outcomes which depend on patient characteristics. If so, statistical controls must be relied on. The statistical adjustment for patient selection of modalities presented in this paper represents an improvement over previous studies comparing various ESRD treatment modalities.

Acknowledgenents - The authors are grateful for the data provided by Dr Philip Held and the Urban Institute, and for comments and assistance from Dr Victor Hawthorne, Ms Sue Eshlemen. seminar participants at the National Center for Health Services Research and Health Care Technology Assessment, and two anonymous reviewers.

\section{REFERENCES}

1. Gornick M, Greenberg JN, Eggers PW. Dobson A. Twenty years of Medicare: covered populations, use of benefits, and program expenditures. Health Care Financ Rev 1985: 7: 1359

2. Rosansky SJ. Eggers PW. Trends in the LS end-stage renal disease population: 1973 1983. Am J Kid Dis 1987:9:9197.

3. Bovbjerg RR, Diamond LH. Held PJ, Pauly MV. Continuous ambulatory peritoneal dialysis: pre liminary evidence in the debate over efficacy and cost. Health Aff 1983: 2:96\%102

4. Carlson DM, Duncan DA. Naessens JM. Johnsoll WL. Hospitalization in dialysis patients. Mayo Clin Proc 1984: 59: 769775

5. Evans RE, Manninen DL, Hart LG, Garrison LP. The Treatment of End-Stage Renal Disease in the U.S.: Selected Findings for the National Kidney Dialysi; and Kidney Transplant Study. Seattle. Wash.: Battelle: Hunall Afrairs Research Center: 1984.

6. Ludbrook A. The cconomics of home care for the treatment of end-stage renal failure. Int $\mathbf{J}$ Technol Assess Health Care 1985; 1: 315324

7. Manninen DL, Evans RW, Hart LG, Garrison LP. A Comparison of the Utilization of Hospital Services Among End-Stage Renal Disease Patients on Four Modalities. Seattle. Wash.: Battelle Human Affairs Research Center; 1983.

8. Prowant BF. Kappel DF. Campbell A. A comparison of inpatient and outpatient medicare allowable charges for ambulatory peritoneal dialysis and center hemodialysis patients: A single center study. Am .I Kid Dis $1986 ; 8: 248252$.

9. Council on Scientific Affairs. Continuous ambulatory peritoneal dialysis. JAMA 1082; 248: 2340-2341 
10. Lameire H. Experience with CAPD as home dialysis. Int J Technol Assess Health Care 1985; 1: 305-314.

11. Stason WB, Barnes BA. The Effectiveness and Costs of Continuous Ambulatory Peritoneal Dialysis (CAPD). U.S. Congress, Office of Technology Assessment OTAHCS-35. Washington, D.C.: U.S. GPO; 1985.

12. Final Notice on New United State Composite Reimbursement Rate Regulations for Prospective Outpatient Maintenance Dialysis Servies. Fed Regist 1986; 51: 29404-29419.

13. Held PJ, Pauly MV. Competition and efficiency in the end stage renal disease program. J Health Econ 1983; 2: $95-118$.

14. Smith DG, Wheeler JRC. Switching and the definition of modality in end-stage renal disease treatment. Med Care 1987; 25: 1168-1178.

15. Simes RJ. Treatment selection for cancer patients: application of statistical decision theory to the treatment of advanced ovarian cancer. J Chron Dis 1985; 38: $171-186$.

16. Starzl TE, et al. A multifactorial system for equitable selection of cadaver kidney recipients. JAMA 1987; 257: 3073-3075.

17. Relman AS, Rennie D. Treatment of end-stage renal disease: free but not equal. N Engl J Med 1980; 303: 996-998.

18. Heckman J. Sample selection bias as a specification error. Econometrica 1979; 47: 239-267.

19. Deber RB, Blinder IN, Carr LM, Barnsley JM. The impact of selected paticnt characteristics of practitioners' treatment recommendations for end-state renal disease. Med Care 1985; 23: 95-109.

20. Welch WP, Frank RG, Diehr P. Health care costs in health maintenance organizations: correcting for selfselection. In: Scheffler, RM, Rossiter L, Eds. Advances in Health Economics and Health Services Research. 1984: Vol. 5, pp. 95-128.

21. Maddala GS. Limited-Dependent and Qualitative Variables in Econometrics. New York: Cambridge University Press; 1983.

22. Lee LF, Trost RP. Technical training and earnings: a polychotomous choice model with selectivity. Rev Fcon Stat 1984; 66: 151-156. 